\title{
Can Social Capital Explain Business Performance in Denmark?
}

\section{Wang, Cong}

2020

Wang , C \& Steiner, PhD , P B 2020 , ' Can Social Capital Explain Business Performance in Denmark? ' , Empirical Economics , vol. 59 , no. 4 , pp. 1-20 . https://doi.org/10.1007/s00181-019-01731-3

http://hdl.handle.net/10138/320290

https://doi.org/10.1007/s00181-019-01731-3

unspecified

acceptedVersion

Downloaded from Helda, University of Helsinki institutional repository.

This is an electronic reprint of the original article.

This reprint may differ from the original in pagination and typographic detail.

Please cite the original version. 


\title{
Social Capital and Firm Performance in Denmark: A Firm-Level Study
}

\author{
Cong Wang*1 and Bodo Steiner**2 \\ *Tasmanian School of Business and Economics, University of Tasmania, Australia \\ **Department of Economics and Management, University of Helsinki, Finland
}

\begin{abstract}
Motivated by the theories that assert a positive link between social capital and firm performance (Hoang and Antoncic (2003), Aldrich and Zimmer (1986)), this paper aims to explore the potential effect of social capital on firm performance by employing a novel approach: to look at social capital environment in a community rather than to focus on the narrow aspect of entrepreneurs' own social network and religious beliefs. Using PCA to aggregate various trust, norm, and network related variables to construct social capital indicators, and measuring firm performance by return on asset, current ratio, solvency ratio and profit margin, this paper has found a positive significant effect of social capital on firm performance in Denmark. These effects are robust to different level of aggregation (sample structure), different sampling year and alternative measure of firm performance.
\end{abstract}

Keywords: Social Capital, Firm Performance, Denmark

JEL Classification: E0, D72, Z10

\section{Introduction}

Current literature increasingly acknowledge that entrepreneurial activity is heavily influenced by network relationships (as a form of social capital) that direct resource flows to entrepreneurs who are somehow better "connected" (see e.g. Hoang and Antoncic (2003), Aldrich and Zimmer (1986)). Adler and Kwon (2002) contend that social capital, or the resources that entrepreneurs may access through their personal networks allows entrepreneurs to identify opportunities, mobilize resources and build legitimacy for their firm. (see also Bhagavatula et al. (2010), Batjargal (2003) and Elfring and Hulsink (2003)). On the other hand, there's a growing strand of literature that also contend that entrepreneurial decisions and activities can also be influenced by religious orientations of the entrepreneurs and the surrounding society (see e.g. Barro and McCleary (2003), Noland (2005) and Tu et al. (2011)).

\footnotetext{
${ }^{1}$ https://www.utas.edu.au/profiles/staff/economics/cong-wang, ORCID: http://orcid.org/0000-0002-6852-5490, ADDRESS: Centenary Building, Sandy Bay Campus, Hobart, Australia

2 https://researchportal.helsinki.fi/en/persons/prof-bodo-steiner-phd, ORCID: https://orcid.org/0000-0002-5357-8856, ADDRESS: P.O. Box 27, FI-00014 FINLAND
} 
Despite the growing interests in this matter in the current literature, consensus has not been reached about the social capital-performance link in the firm context. According to at least Maurer and Ebers (2006), Stuart and Sorensen (2007) and Stam et al. (2014), there exists conflicting perspectives regarding the specific network properties that constitute social capital. While some have focused on network structure (see e.g. Stam (2010)), others have considered the strength of entrepreneur's network relationships or the resources held by their network contacts. (see e.g. Batjargal (2003), McEvily and Zaheer (1999)). Disagreement looms largely over whether a sparse or cohesive network, weak or strong ties, and diverse or homogenous network could potentially promote firm growth. (Renzulli et al. (2000)). While some have argued that cohesive, strong-tie networks are conducive for new firms (Hite and Hesterly (2001), others have contended that diverse, weak -tie networks are favorable at the early stages of firm development. (Elfring and Hulsink (2007)). Martinez and Aldrich (2011) also points out that ambiguity exists about the temporal contingencies that govern when certain forms of social capital are most beneficial for firm performance.

Motivated by the above inconclusive results from the current literature, this paper aims to provide a comprehensive analysis on the role of social capital in firm performance in Denmark by examining a broader social capital environment rather than focusing on the narrow aspect of entrepreneurs' personal networks as the sole measure of social capital that might matter for firm performance. We measure social capital in the context of trust, norm (civic attitude) and two different network types as defined by Putnam et al. (1993) and Olson (1982) in 14 Danish regions with over 1,000 postal locations, and investigate the potential effect of social capital endowment on firm performance (measured by various financial and innovation performance indicators such as return on assets, solvency ratio, current ratio and profit margin) in these regions. The underlying hypothesis is that businesses, regardless large or small, perform better by doing business in a better social capital environment with high local trust, good civil norms and growth enhancing networks.

This paper therefore contributes to the current literature in three ways: (1) it bridges the gap between two related strands of literature by linking the macro type of analysis on the effect of social capital on macroeconomic growth (see e.g. Knack and Keefer (1997)) to the micro level analysis on the effect of local social capital (previously only in the form of entrepreneur's personal networks) on firm performance and growth. (2) In addition, this paper also addresses regional imbalance/inequality in the context of both social capital and firm development by including urban and border dummies to explore the potential urban-rural, border-non border region divide in terms of firm performance. 
Using PCA to combine various trust, norm and network related variables from the Danish value studies, this study constructs its own social capital variables for Denmark and generally found a strong positive effect of social capital on firm performance as measured by return on asset, current ratio, solvency ratio and profit margin. The remainder of this paper is structured as follows: Section 2 reviews the literature, Section 3 perform firm-level analysis and carry out robustness checks to different level of aggregation, different sampling year and alternative measure of firm performance. Section 4 concludes.

\section{Literature Review}

\section{Analysis}

\subsection{Data and Descriptive Statistics}

Following the approach introduced in Wang and Steiner (2015), Social capital is measured by three components: trust, norm and network. The data are obtained from the 1999 and 2008 waves of Danish Value Studies (DVS) ${ }^{3}$. The trust component of social capital measures how much one person generally trusts other people in one's neighborhood, and how much confidence one has in private, public and political institutions. We use the first principal component analysis (PCA) to combine the percentage of respondents that give a positive answer to a trust-related question and form the variable trust (domestic), if the trust-related questions concerning only domestic institutions and organizations, and trust (foreign) if the questions concerning foreign institutions and organizations. Norm is measured by the PCA combination of the percentage of respondents that do not justify various generally socially unacceptable behaviors, such as cheating on tax, lying in own interest, accepting a bribe and drunk driving etc.

The network component of social capital is measured in three ways: first, we use PCA to combine the membership in all organizations surveyed in the value studies to form the network (all group) variable. Second, following the approach introduced in Wang and Steiner (2015), we differentiate two types of organizations: "Putnam" and "Olson". The "Putnam" organizations (see Putnam et al. (1993)) are religious, education, arts and sports organizations that do not exclude horizontal integration, i.e. people across these organizations can bond and social well without confliction of interests. On the contrary, the "Olson" organizations (see Olson (1982)) are special interests groups that prevent people across organizations to communicate and cooperate in an effective manner, the examples are labor unions,

\footnotetext{
${ }^{3}$ There're 1,023 and 1,507 individuals being surveyed in the 1999 and 2009 waves of Danish Value Studies respectively. Regional identifier (Amter) and Postal codes are available for regional level social capital aggregation, due to the extremely low observations for each postal code area (1 to 2 at most), to meaningfully calculate regional level social capital, we decide to aggregate individual values to the 14-Amter regional level. Therefore firms located in the same region (Amter) share the same social capital environment with same trust, norm and network societal values.
} 
political parties and professional organizations. The PCA combination of membership in "Putnam" organizations forms the variable Network (Putnam), and the PCA combination of membership in "Olson" organizations forms the variable Network (Olson). The detailed survey questions and PCA coefficient used to construct the above social capital variables can be found in Table A1 in the appendix.

Firm performance is measured by three firm-level key financial performance indicators: return on assets, current ratio and solvency ratio for all Danish firms ${ }^{4}$. We also include profit margin in the robustness check. The data are obtained from the Orbis and Amadeus database. Return on asset is defined as the ratio of net income to total asset. Current ratio is defined as the ratio of current asset to current liability. Solvency ratio is defined as the ratio of net income plus depreciation to short term and long term liability. Profit margin is defined as the ratio of net income to total revenue.

We control firm size, regional health status, high education status, urban dummies, and border dummies in the relationship concerning social capital and firm performance. Firm size is measured by total asset of a firm, obtained from Orbis. Health status is the percentage of respondents identifies themselves as being in "excellent health" and "good health" in a given Danish Amter, High education is measured by the percentage of respondents identify themselves as having completed "first stage tertiary education" in a given Danish region. Both health and education variables are obtained from the DVS. The urban dummy equals 1 if an underlying region has a degree of urbanization value greater than 4 out of a 1 to 6 point scale as defined by the DVS, and 0 other wise. The Border dummy equals 1 if a firm belongs to the Southern Jutland region, and 0 otherwise.

Table 1 gives summary statistics and definitions for all variables involved in the estimation of the 2008 firm-level sample. A close look at the means and standard deviations shows low variability in the social capital and protestant religious groups and high variability in firm performance indicators and religious groups other than protestant. These are in line with expectations, as Denmark is a rather "universal" small country with similar culture and religious beliefs across regions. On the other hand, the data shows firm types (indicated by total asset) and performances are diverse. This seems to suggest if any robust effects of social capital on firm performance can be found in a sample like this, these effects must be really strong as tiny variations in social capital can cause huge variations in firm performance. A correlation between all variables can also be found in Table A2 in the appendix.

\section{Table 1: Summary Statistics for the 2008 Firm-Level Sample}

\footnotetext{
${ }^{4}$ There're in total 739,588 Danish firms registered with Orbis and Amadeus from the year 2000 to 2013. Observations for return on assets, current ratio and solvency ratio are 178,886, 192,637 and 200,427 respectively. Profit margin has only 20,617 observations, therefore is used as a robustness check.
} 


\begin{tabular}{|c|c|c|c|c|c|c|}
\hline Variable & Definition & Obs. & Mean & $\begin{array}{l}\text { Std. } \\
\text { Dev. }\end{array}$ & Min & Max \\
\hline $\begin{array}{c}\text { Trust } \\
\text { (domestic) }\end{array}$ & $\begin{array}{l}\text { PCA combination of domestically trust- } \\
\text { related indicators measured in percentage of } \\
\text { respondents given positive answers in a given } \\
\text { Danish region (14-Amter) }\end{array}$ & 695646 & 65.62 & 3.16 & 59.40 & 72.58 \\
\hline Trust (foreign) & $\begin{array}{l}\text { PCA combination of foreign trust-related } \\
\text { indicators measured in percentage of } \\
\text { respondents given positive answers in a given } \\
\text { Danish region (14-Amter) }\end{array}$ & 695646 & 56.92 & 3.73 & 47.74 & 62.45 \\
\hline Norm & $\begin{array}{l}\text { PCA combination of norms-related indicators } \\
\text { measured in percentage of respondents given } \\
\text { positive answers in a given Danish region (14- } \\
\text { Amter) }\end{array}$ & 695646 & 52.60 & 3.97 & 40.48 & 60.47 \\
\hline $\begin{array}{l}\text { Network (all } \\
\text { groups) }\end{array}$ & $\begin{array}{l}\text { PCA combination of Network(all groups)- } \\
\text { related indicators measured in percentage of } \\
\text { respondents given positive answers in a given } \\
\text { Danish region (14-Amter) }\end{array}$ & 695646 & 29.09 & 1.37 & 26.57 & 34.06 \\
\hline $\begin{array}{c}\text { Network } \\
\text { (Putnam group) }\end{array}$ & $\begin{array}{l}\text { PCA combination of Network(Putnam group)- } \\
\text { related indicators measured in percentage of } \\
\text { respondents given positive answers in a given } \\
\text { Danish region (14-Amter) }\end{array}$ & 695646 & 12.10 & 1.10 & 10.05 & 15.59 \\
\hline $\begin{array}{l}\text { Network (Olson } \\
\text { group) }\end{array}$ & $\begin{array}{l}\text { PCA combination of Network(Olson group)- } \\
\text { related indicators measured in percentage of } \\
\text { respondents given positive answers in a given } \\
\text { Danish region (14-Amter) }\end{array}$ & 695646 & 23.12 & 1.20 & 20.52 & 27.68 \\
\hline $\begin{array}{l}\text { Firm size (total } \\
\text { assets) }\end{array}$ & Total asset of a firm as of 2008 & 217085 & 27630.45 & 2311946 & -51.89 & $6.64 \mathrm{E}+08$ \\
\hline Health status & $\begin{array}{l}\text { Percentage of respondents identify } \\
\text { themselves as being in "excellent health" and } \\
\text { "good health" in a given Danish region (14- } \\
\text { Amter) }\end{array}$ & 695646 & 79.30 & 4.09 & 71.15 & 84.87 \\
\hline High education & $\begin{array}{l}\text { Percentage of resopndents identify } \\
\text { themselves as have completed "first stage } \\
\text { tertiary education" in a given Danish region } \\
\text { (14-Amter) }\end{array}$ & 695646 & 38.45 & 10.95 & 14.29 & 53.97 \\
\hline Urban & $\begin{array}{l}\text { Dummy variable that equals } 1 \text { if a given } \\
\text { Danish region (14-Amter) has a "degree of } \\
\text { urbanization" value greater than } 4 \text { (in a } 1 \text { to } 6 \\
\text { scale) and } 0 \text { otherwise. }\end{array}$ & 695646 & 0.54 & 0.48 & 0 & 1 \\
\hline Border & $\begin{array}{l}\text { Dummy variable that equals } 1 \text { when a firm is } \\
\text { located in the Southern Jutland region } \\
\text { (Danish-German border region), and } 0 \\
\text { otherwise. }\end{array}$ & 695646 & 0.06 & 0.23 & 0 & 1 \\
\hline Return on & net income & 178866 & 12.56 & 96.35 & - & 995.74 \\
\hline Assets & $\overline{\text { total asset }}$ & & & & 998.75 & \\
\hline Current Ratio & $\frac{\text { current asset }}{\text { current liability }}$ & 192637 & 6.14 & 14.03 & 0 & 100 \\
\hline
\end{tabular}




\begin{tabular}{ccccccc}
\hline Solvency Ratio & net income + depreciation & 200427 & 50.16 & 41.73 & -100 & 100 \\
\cline { 2 - 5 } & short term liability + long term liabitlity & & & & & \\
Profit Margin & $\frac{n e t \text { income }}{\text { total revenue }}$ & 20617 & 11.01 & 33.39 & -100 & 100
\end{tabular}

Notes: For specific sub-indicators (original questions used in the 2008 Danish value studies surveys), see Table A1 in the appendix. Firms located in the same region (one of 14) are facing the same level of social capital (trust, norm and network), resident health environment (health status), education level (high education) and religious environment (Catholic, Protestant, Jews, Muslim and Buddhist).

\subsection{Estimation (firm-level regression)}

This section attempts to estimate the following equation:

$$
\text { firm perfromance }=\alpha_{0}+\alpha_{1} \text { social capital }+\alpha_{2} X+u
$$

Where the firm performance variables are return on assets, current ratio and solvency ratio; social capital variables are Trust (domestic), Trust (foreign), Norm, Network(all group), Network (Putnam), and Network (Olson); $X$ is a set a control variables that include firm size, health status, high education, urban dummies and border dummies. $u$ is the error term.

The OLS estimations with heteroskedasticity-consistent standard errors presented in Table 2 test the effect of social capital on firm performance. From Table 2, Columns (1) to (4) show the results on return on assets as a measure of firm performance, with Columns (5) to (8) and Columns (9) to (12) showing the results for current ratio and solvency ratio respectively. Individually, all three aspects of social capital (trust, norm and network) show strong significant influence on firm performance, although comparatively speaking, trust and network stand out, with the effect of norm being considerably weaker. The coefficient on Trust and Network suggest that a 1 unit increase in trust and membership in organizations boost return on asset by $1.05 \%$ to $1.6 \%$, while a 1 unit increase in social norm boosts return on asset by about $0.2 \%$. When including all regressors (i.e. two trust measures, norm and three network measures) into the regression, trust and network continue to show strong results, whilst norm becomes insignificant. Given the strong correlations between our social capital variables (see Table A2), multicollinearity could be the potential cause of the insignificance of norm. The consistent positive effect of social capital on firm growth is evident by looking at all three firm growth measures.

Trust in domestic institutions seems matter more for firm growth than trust in foreign institutions. This is evident when exploring all three measures of firm performances. Cross comparing these three measures shows that the magnitude of the effect of social capital on return on assets and solvency ratio 
seem to be generally stronger than the effect on current ratio. Social capital seems to matter more for long-term liquidity (solvency ratio) than short-term liquidity (current ratio). Our results from networks suggest that all networking types are important for firm growth. The Putnam group of networking is often seen as being conducive to general economic growth (Putnam (1993)), while the Olson grouping (Olson (1982)) is generally seen as being growth hindering, since this type of grouping represents special interests group that often causes between-group conflicts. These however should not be viewed as contradicting our results on firm growth, as firm growth and general macroeconomic growth are different. Firms are micro-entities that can be influenced by specific types of networks, each special interests group can positively influence a specific type of firm. Therefore the Olson group does not generate the type of between-group conflict when dealing with specific firms as opposed to when dealing with the general economy. A special interests group's particular interest can coincide with a particular type of firm's objectives. Hence, we observe the universal positive effect of Olson, Putnam and All-group networking on firm performance. In general, our findings on the positive effect of social capital (especially networking) on firm performance is in line with findings from Aldrich and Zimmer (1986), Hoang and Antoncic (2003) and Stam (2010).

Moreover, turning to the control variables, as expected, the firm size (measured by total asset) control turns out to be insignificant for firm performance, suggesting that small firms have just the same opportunity to be successful as their large counterparts. Both high education status (human capital) and good health status turn out to be good for firm performance. At least in the case of Denmark, the urban advantage exists for firm performance as we saw a skewed resource distribution towards the urban area in Denmark. The Danish border region seems to do worse in terms of firm performance, as this southern Jutland region of Denmark is generally an underdeveloped area. The F-statistics and their p-values suggest the variables included in the regression are jointly significant in all cases, and the Rsquares suggest anywhere between $9 \%$ and $27 \%$ of the model is explained.

\subsection{Robustness Checks}

We perform three types of robustness checks. First, to address the natural limitation of our social capital data (aggregated from 1,000+ respondents to 14 region level), we aggregate the firm level performance data onto three different levels (1) 14-region level, effectively, the average firm performance per region corresponds to its regional social capital value. (2) region-industry level, in which a particular industry within a particular region is treated as one observation, so that we differentiate industries within a particular region to take into account industry heterogeneity. (3) postal 
code-industry level, which is similar to the region-industry aggregation, except that we identify a particular industry within a specific postal code as one observation. The purpose of these 
Table 2: Social Capital and Firm Performance (OLS firm-level regressions)

\begin{tabular}{|c|c|c|c|c|c|c|c|c|c|c|c|c|}
\hline \multicolumn{13}{|c|}{ Firm-Level regressions (all variables measured in 2008) } \\
\hline & (1) & $(2)$ & (3) & (4) & (5) & (6) & (7) & (8) & (9) & (10) & (11) & $(12)$ \\
\hline & $\begin{array}{c}\text { Return } \\
\text { on Assets }\end{array}$ & $\begin{array}{c}\text { Return on } \\
\text { Assets }\end{array}$ & $\begin{array}{c}\text { Return on } \\
\text { Assets }\end{array}$ & $\begin{array}{c}\text { Return on } \\
\text { Assets }\end{array}$ & $\begin{array}{c}\text { Current } \\
\text { Ratio }\end{array}$ & $\begin{array}{l}\text { Current } \\
\text { Ratio }\end{array}$ & $\begin{array}{l}\text { Current } \\
\text { Ratio }\end{array}$ & $\begin{array}{c}\text { Current } \\
\text { Ratio }\end{array}$ & $\begin{array}{c}\text { Solvency } \\
\text { Ratio }\end{array}$ & $\begin{array}{c}\text { Solvency } \\
\text { Ratio }\end{array}$ & $\begin{array}{c}\text { Solvency } \\
\text { Ratio }\end{array}$ & $\begin{array}{c}\text { Solvency } \\
\text { Ratio }\end{array}$ \\
\hline \multicolumn{13}{|c|}{ Social Capital and Religion Variables } \\
\hline $\begin{array}{c}\text { Trust } \\
\text { (domestic) }\end{array}$ & $\begin{array}{c}1.291 * * \\
(2.43)\end{array}$ & & & $\begin{array}{c}5.612 * * \\
(2.42)\end{array}$ & $\begin{array}{c}0.308 * * \\
(2.13)\end{array}$ & & & $\begin{array}{c}0.693^{*} \\
(1.70)\end{array}$ & $\begin{array}{c}1.390 * * * \\
(3.89)\end{array}$ & & & $\begin{array}{c}0.922 * \\
(1.95)\end{array}$ \\
\hline Trust (foreign) & $\begin{array}{c}0.862 * \\
(1.92)\end{array}$ & & & $\begin{array}{c}1.682 * \\
(1.68)\end{array}$ & $\begin{array}{c}0.244^{*} \\
(1.72)\end{array}$ & & & $\begin{array}{c}0.447 * * \\
(2.47)\end{array}$ & $\begin{array}{c}1.367^{* * * *} \\
(2.96)\end{array}$ & & & $\begin{array}{c}0.915 * * \\
(2.09)\end{array}$ \\
\hline Norm & & $\begin{array}{c}0.493 * * \\
(2.00)\end{array}$ & & $\begin{array}{l}-1.824 \\
(-1.51)\end{array}$ & & $\begin{array}{c}0.127^{* * *} \\
(3.61)\end{array}$ & & $\begin{array}{l}0.113 \\
(0.54)\end{array}$ & & $\begin{array}{c}0.449 * * * \\
(4.49)\end{array}$ & & $\begin{array}{c}1.116 * * \\
(2.22)\end{array}$ \\
\hline $\begin{array}{l}\text { Network (all- } \\
\text { group) }\end{array}$ & & & $\begin{array}{c}2.931 * * * \\
(3.11)\end{array}$ & $\begin{array}{c}3.588 * * * \\
(3.01)\end{array}$ & & & $\begin{array}{c}0.129 * * \\
(2.41)\end{array}$ & $\begin{array}{c}1.734^{*} \\
(1.71)\end{array}$ & & & $\begin{array}{l}0.691 * \\
(1.77)\end{array}$ & $\begin{array}{l}-1.978 \\
(-0.34)\end{array}$ \\
\hline $\begin{array}{l}\text { Network } \\
\text { (Putnam) }\end{array}$ & & & & $\begin{array}{c}6.629 * * \\
(2.26)\end{array}$ & & & & $\begin{array}{c}1.159 * * * \\
(2.90)\end{array}$ & & & & $\begin{array}{c}2.687 * * \\
(2.47)\end{array}$ \\
\hline $\begin{array}{c}\text { Network } \\
\text { (Olson) }\end{array}$ & & & & $\begin{array}{c}3.552 * * * \\
(3.00)\end{array}$ & & & & $\begin{array}{l}2.932 \\
(0.84)\end{array}$ & & & & $\begin{array}{l}0.697 \\
(0.08)\end{array}$ \\
\hline \multicolumn{13}{|l|}{ Control Variables } \\
\hline $\begin{array}{c}\text { Firm size } \\
\text { (total assets) }\end{array}$ & $\begin{array}{c}4.72 \mathrm{e}-07 \\
(1.04)\end{array}$ & $\begin{array}{c}4.09 e-07 \\
(0.58)\end{array}$ & $\begin{array}{c}3.65 e-07 \\
(0.20)\end{array}$ & $\begin{array}{c}2.11 \mathrm{e}-07 \\
(1.19)\end{array}$ & $\begin{array}{c}-2.67 e-07 \\
(-0.81)\end{array}$ & $\begin{array}{c}-2.70 e-08 \\
(-0.27)\end{array}$ & $\begin{array}{c}-2.72 \mathrm{e}-08 \\
(-1.61)\end{array}$ & $\begin{array}{c}-2.57 e-07 \\
(-0.45)\end{array}$ & $\begin{array}{c}-7.45 e-07 \\
(-1.41)\end{array}$ & $\begin{array}{c}-1.48 \mathrm{e}-07 \\
(-0.88)\end{array}$ & $\begin{array}{c}-6.11 e-08 \\
(-1.06)\end{array}$ & $\begin{array}{c}-7.21 e-07 \\
(-1.37)\end{array}$ \\
\hline Health status & $\begin{array}{l}-0.099 \\
(-0.24)\end{array}$ & $\begin{array}{l}0.111 \\
(0.32)\end{array}$ & $\begin{array}{l}-0.182 \\
(-0.58)\end{array}$ & $\begin{array}{l}-0.182 \\
(-0.16)\end{array}$ & $\begin{array}{l}-0.297 \\
(-1.23)\end{array}$ & $\begin{array}{c}0.099 * \\
(1.73)\end{array}$ & $\begin{array}{c}0.083^{* * *} \\
(2.70)\end{array}$ & $\begin{array}{c}0.521^{* * *} \\
(2.56)\end{array}$ & $\begin{array}{c}1.132 * * \\
(2.04)\end{array}$ & $\begin{array}{c}0.363^{* *} \\
(2.21)\end{array}$ & $\begin{array}{l}0.154 \\
(0.76)\end{array}$ & $\begin{array}{l}-0.477 \\
(-0.95)\end{array}$ \\
\hline $\begin{array}{c}\text { High } \\
\text { education }\end{array}$ & $\begin{array}{l}-0.220 \\
(-1.23)\end{array}$ & $\begin{array}{l}-0.071 \\
(-0.38)\end{array}$ & $\begin{array}{l}0.014 \\
(0.09)\end{array}$ & $\begin{array}{l}-0.805 \\
(-1.20)\end{array}$ & $\begin{array}{c}0.609 * * * \\
(3.30)\end{array}$ & $\begin{array}{c}0.070 * * * \\
(4.54)\end{array}$ & $\begin{array}{c}0.027^{*} \\
(1.82)\end{array}$ & $\begin{array}{c}0.938 * * * \\
(6.81)\end{array}$ & $\begin{array}{c}2.075^{* *} \\
(4.70)\end{array}$ & $\begin{array}{c}0.187^{* * *} \\
(4.13)\end{array}$ & $\begin{array}{l}-0.093 \\
(-0.92)\end{array}$ & $\begin{array}{c}2.291^{* * *} \\
(7.15)\end{array}$ \\
\hline Urban & $\begin{array}{l}-3.702 \\
(-1.00)\end{array}$ & $\begin{array}{l}-2.497 \\
(-0.66)\end{array}$ & $\begin{array}{l}-3.165 \\
(-0.88)\end{array}$ & $\begin{array}{c}4.842 * * \\
(2.32)\end{array}$ & $\begin{array}{c}7.008^{* * *} \\
(2.80)\end{array}$ & $\begin{array}{c}2.151^{* * *} \\
(4.27)\end{array}$ & $\begin{array}{l}-0.359 \\
(-1.32)\end{array}$ & $\begin{array}{c}9.408^{* * *} \\
\quad(3.06)\end{array}$ & $\begin{array}{c}21.279 * * * \\
(3.57)\end{array}$ & $\begin{array}{c}7.047^{* * *} \\
(4.90)\end{array}$ & $\begin{array}{l}-0.515 \\
(-0.29)\end{array}$ & $\begin{array}{c}33.658^{* * *} \\
(4.51)\end{array}$ \\
\hline Border & $\begin{array}{l}4.746 \\
(0.70)\end{array}$ & $\begin{array}{c}-11.399^{*} \\
(-1.66)\end{array}$ & $\begin{array}{l}0.742 \\
(0.12)\end{array}$ & $\begin{array}{l}-2.912 \\
(-0.05)\end{array}$ & $\begin{array}{l}-0.779 \\
(-0.30)\end{array}$ & $\begin{array}{c}-2.467 * * * \\
(-2.98)\end{array}$ & $\begin{array}{l}-0.144 \\
(-0.41)\end{array}$ & $\begin{array}{l}-1.723 \\
(-0.72)\end{array}$ & $\begin{array}{c}-11.575^{*} \\
(-1.89)\end{array}$ & $\begin{array}{c}-8.273 * * * \\
(-3.51)\end{array}$ & $\begin{array}{l}1.266 \\
(0.55)\end{array}$ & $\begin{array}{c}-6.519 * * * \\
(-4.32)\end{array}$ \\
\hline \multicolumn{13}{|c|}{ Diagnostic Statistics } \\
\hline $\mathrm{N}$ & 158185 & 158185 & 158185 & 158185 & 169398 & 169398 & 169398 & 169398 & 177034 & 177034 & 177034 & 177034 \\
\hline $\mathrm{R}-\mathrm{sq}$ & 0.12 & 0.14 & 0.10 & 0.21 & 0.16 & 0.14 & 0.09 & 0.22 & 0.15 & 0.13 & 0.08 & 0.27 \\
\hline $\begin{array}{c}\text { F-statistic } \\
\text { (p-value) }\end{array}$ & $\begin{array}{c}13.30 \\
(0.000)\end{array}$ & $\begin{array}{c}13.68 \\
(0.000)\end{array}$ & $\begin{array}{c}14.24 \\
(0.000)\end{array}$ & $\begin{array}{c}16.54 \\
(0.000)\end{array}$ & $\begin{array}{c}14.26 \\
(0.000)\end{array}$ & $\begin{array}{c}10.77 \\
(0.000)\end{array}$ & $\begin{array}{c}10.10 \\
(0.000)\end{array}$ & $\begin{array}{c}68.07 \\
(0.000)\end{array}$ & $\begin{array}{c}8.21 \\
(0.000)\end{array}$ & $\begin{array}{c}22.29 \\
(0.000)\end{array}$ & $\begin{array}{c}15.90 \\
(0.000)\end{array}$ & $\begin{array}{c}80.21 \\
(0.000)\end{array}$ \\
\hline
\end{tabular}

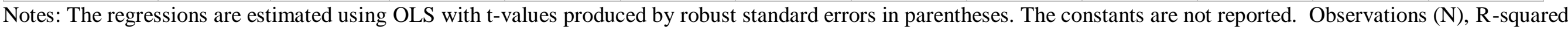
$(\mathrm{R}-\mathrm{sq}), \mathrm{F}$-statistics and the associated p-values are reported. Significance at the $1 \%, 5 \%$ and $10 \%$ levels are denoted respectively by $* * *, * *$ and $*$. 
Table 3: Social Capital and Firm Performance: Robustness to Different Levels of Aggregation

\begin{tabular}{|c|c|c|c|c|c|c|c|c|c|}
\hline & \multicolumn{3}{|c|}{ Regional Level Sample } & \multicolumn{3}{|c|}{ Region-Industry Level Sample } & \multicolumn{3}{|c|}{ Postal Code-Industry Level Sample } \\
\hline & (1) & $(2)$ & (3) & (4) & $(5)$ & $(6)$ & (7) & (8) & (9) \\
\hline & $\begin{array}{c}\text { Return on } \\
\text { Assets }\end{array}$ & $\begin{array}{c}\text { Current } \\
\text { Ratio }\end{array}$ & Solvency Ratio & $\begin{array}{c}\text { Return on } \\
\text { Assets }\end{array}$ & Current Ratio & Solvency Ratio & Return on Assets & Current Ratio & Solvency Ratio \\
\hline \multicolumn{10}{|c|}{ Social Capital and Religion Variables } \\
\hline $\begin{array}{c}\text { Trust } \\
\text { (domestic) }\end{array}$ & $\begin{array}{c}0.399 * \\
(3.72)\end{array}$ & $\begin{array}{c}0.291 * \\
(3.69)\end{array}$ & $\begin{array}{c}0.192 * \\
(3.98)\end{array}$ & $\begin{array}{c}1.077^{* * *} \\
(3.56)\end{array}$ & $\begin{array}{c}\text { 5.998* } \\
(1.78)\end{array}$ & $\begin{array}{c}-17.808 \\
(-1.09)\end{array}$ & $\begin{array}{l}1.991 * \\
(1.65)\end{array}$ & $\begin{array}{c}1.864 * * * \\
(6.54)\end{array}$ & $\begin{array}{c}2.165^{* * *} \\
(4.65)\end{array}$ \\
\hline Trust (foreign) & $\begin{array}{l}\text { Omitted (see } \\
\text { notes below) }\end{array}$ & $\begin{array}{l}\text { Omitted (see } \\
\text { notes below) }\end{array}$ & $\begin{array}{l}\text { Omitted (see } \\
\text { notes below) }\end{array}$ & $\begin{array}{c}0.508^{* * *} \\
(4.32)\end{array}$ & $\begin{array}{c}1.096 * * * \\
(3.20)\end{array}$ & $\begin{array}{c}6.841 * * * \\
(4.52)\end{array}$ & $\begin{array}{l}1.089 \\
(1.27)\end{array}$ & $\begin{array}{l}0.154 \\
(1.17)\end{array}$ & $\begin{array}{c}1.401 * * * \\
(2.76)\end{array}$ \\
\hline Norm & $\begin{array}{l}0.981 \\
(2.15)\end{array}$ & $\begin{array}{c}0.052 * * \\
(7.88)\end{array}$ & $\begin{array}{c}0.124 * * \\
(4.52)\end{array}$ & $\begin{array}{c}2.230 * * * \\
(3.23)\end{array}$ & $\begin{array}{l}1.520^{*} \\
(1.65)\end{array}$ & $\begin{array}{l}4.699 \\
(1.19)\end{array}$ & $\begin{array}{c}1.773 * * \\
(2.37)\end{array}$ & $\begin{array}{c}1.037^{* * *} \\
(5.66)\end{array}$ & $\begin{array}{c}1.976^{* * *} \\
(7.60)\end{array}$ \\
\hline $\begin{array}{c}\text { Network (all- } \\
\text { group) }\end{array}$ & $\begin{array}{c}\text { 7.195*** } \\
(12.13)\end{array}$ & $\begin{array}{c}11.792 \\
(0.98)\end{array}$ & $\begin{array}{l}1.792 \\
(0.45)\end{array}$ & $\begin{array}{r}-12.587 \\
(-0.24)\end{array}$ & $\begin{array}{c}14.911 * * \\
(2.32)\end{array}$ & $\begin{array}{c}7.306 * * \\
(2.73)\end{array}$ & $\begin{array}{l}4.290 \\
(0.61)\end{array}$ & $\begin{array}{c}7.422 * * * \\
(5.90)\end{array}$ & $\begin{array}{l}2.165 \\
(0.57)\end{array}$ \\
\hline $\begin{array}{l}\text { Network } \\
\text { (Putnam) }\end{array}$ & $\begin{array}{l}\text { Omitted (see } \\
\text { notes below) }\end{array}$ & $\begin{array}{l}\text { Omitted (see } \\
\text { notes below) }\end{array}$ & $\begin{array}{l}\text { Omitted (see } \\
\text { notes below) }\end{array}$ & $\begin{array}{c}2.362 * * * \\
(3.20)\end{array}$ & $\begin{array}{c}1.663^{*} \\
(1.83)\end{array}$ & $\begin{array}{l}-2.882 \\
(-0.73)\end{array}$ & $\begin{array}{c}2.073 * \\
(1.90)\end{array}$ & $\begin{array}{c}7.269 * * * \\
(6.33)\end{array}$ & $\begin{array}{c}2.108 * * * \\
(5.65)\end{array}$ \\
\hline $\begin{array}{l}\text { Network } \\
\text { (Olson) }\end{array}$ & $\begin{array}{l}\text { Omitted (see } \\
\text { notes below }\end{array}$ & $\begin{array}{l}\text { Omitted (see } \\
\text { notes below) }\end{array}$ & $\begin{array}{l}\text { Omitted (see } \\
\text { notes below) }\end{array}$ & $\begin{array}{c}-36.021 \\
(-1.24)\end{array}$ & $\begin{array}{c}7.614^{* *} \\
(2.03)\end{array}$ & $\begin{array}{c}3.304^{* *} \\
(2.10)\end{array}$ & $\begin{array}{c}7.334 * \\
(1.89)\end{array}$ & $\begin{array}{c}3.841 * * * \\
(4.66)\end{array}$ & $\begin{array}{c}9.833 * * * \\
(5.56)\end{array}$ \\
\hline \multicolumn{10}{|l|}{ Control Variables } \\
\hline $\begin{array}{c}\text { Firm size } \\
\text { (total assets) }\end{array}$ & $\begin{array}{c}1.17 e-07 \\
(0.17)\end{array}$ & $\begin{array}{c}-6.22 \mathrm{e}-08 \\
(-2.98)\end{array}$ & $\begin{array}{c}-2.61 e-07 \\
(-2.73)\end{array}$ & $\begin{array}{c}-2.54 \mathrm{e}-09 \\
(-0.12)\end{array}$ & $\begin{array}{c}2.74 \mathrm{e}-08 \\
(0.34)\end{array}$ & $\begin{array}{c}1.60 \mathrm{e}-07 \\
(0.74)\end{array}$ & $\begin{array}{c}1.30 \mathrm{e}-07 \\
(1.48)\end{array}$ & $\begin{array}{c}-1.09 e-07 \\
(-0.48)\end{array}$ & $\begin{array}{c}2.20 \mathrm{e}-07 \\
(1.48)\end{array}$ \\
\hline Health status & $\begin{array}{l}-0.195 \\
(-1.11)\end{array}$ & $\begin{array}{c}0.152 * \\
(4.42)\end{array}$ & $\begin{array}{c}0.402^{* *} \\
(4.51)\end{array}$ & $\begin{array}{c}4.577^{* *} \\
(2.17)\end{array}$ & $\begin{array}{c}5.209^{*} \\
(1.87)\end{array}$ & $\begin{array}{c}9.348^{*} \\
(1.65)\end{array}$ & $\begin{array}{c}7.004^{* *} \\
(2.12)\end{array}$ & $\begin{array}{c}12.730 * * * \\
(6.12)\end{array}$ & $\begin{array}{c}18.605^{* * *} \\
(7.76)\end{array}$ \\
\hline $\begin{array}{c}\text { High } \\
\text { education }\end{array}$ & $\begin{array}{l}\text { Omitted (see } \\
\text { notes below }\end{array}$ & $\begin{array}{l}\text { Omitted (see } \\
\text { notes below) }\end{array}$ & $\begin{array}{l}\text { Omitted (see } \\
\text { notes below) }\end{array}$ & $\begin{array}{c}2.095^{* * *} \\
(3.28)\end{array}$ & $\begin{array}{l}-1.281 \\
(-1.49)\end{array}$ & $\begin{array}{l}4.536 \\
(1.23)\end{array}$ & $\begin{array}{l}-0.996 \\
(-0.89)\end{array}$ & $\begin{array}{c}1.443^{* * *} \\
(6.40)\end{array}$ & $\begin{array}{l}-1.395 \\
(-0.77)\end{array}$ \\
\hline Urban & $\begin{array}{l}\text { Omitted (see } \\
\text { notes below }\end{array}$ & $\begin{array}{l}\text { Omitted (see } \\
\text { notes below) }\end{array}$ & $\begin{array}{l}\text { Omitted (see } \\
\text { notes below) }\end{array}$ & $\begin{array}{c}3.157^{* * * *} \\
(3.84)\end{array}$ & $\begin{array}{c}15.923 \\
(1.45)\end{array}$ & $\begin{array}{c}-47.657 \\
(-1.01)\end{array}$ & $\begin{array}{c}4.575^{* *} \\
(2.05)\end{array}$ & $\begin{array}{c}-34.515 \\
(-0.00)\end{array}$ & $\begin{array}{c}-48.813 \\
(-1.19)\end{array}$ \\
\hline Border & $\begin{array}{c}-13.572 * * \\
(-5.33)\end{array}$ & $\begin{array}{l}0.877 \\
(0.85)\end{array}$ & $\begin{array}{l}6.194 \\
(1.72)\end{array}$ & $\begin{array}{c}-6.596 * * * \\
(-3.82)\end{array}$ & $\begin{array}{c}-33.759 \\
(-1.45)\end{array}$ & $\begin{array}{c}100.499 \\
(1.00)\end{array}$ & $\begin{array}{c}-11.258^{* *} \\
(-2.04)\end{array}$ & $\begin{array}{c}-10.466 * * * \\
(-6.16)\end{array}$ & $\begin{array}{c}135.008 \\
(0.05)\end{array}$ \\
\hline \multicolumn{10}{|c|}{ Diagnostic Statistics } \\
\hline $\mathrm{N}$ & 14 & 14 & 14 & 2936 & 2882 & 2989 & 33022 & 39516 & 39995 \\
\hline $\mathrm{R}-\mathrm{sq}$ & 0.99 & 0.99 & 0.99 & 0.11 & 0.19 & 0.17 & 0.32 & 0.29 & 0.22 \\
\hline $\begin{array}{c}\text { F-statistic } \\
\text { (p-value) }\end{array}$ & $\begin{array}{c}37.58 \\
(0.000)\end{array}$ & $\begin{array}{c}39.37 \\
(0.000)\end{array}$ & $\begin{array}{c}40.18 \\
(0.000)\end{array}$ & $\begin{array}{c}10.62 \\
(0.000)\end{array}$ & $\begin{array}{c}13.21 \\
(0.000)\end{array}$ & $\begin{array}{c}14.04 \\
(0.000)\end{array}$ & $\begin{array}{c}32.74 \\
(0.000)\end{array}$ & $\begin{array}{c}16.97 \\
(0.000)\end{array}$ & $\begin{array}{c}17.88 \\
(0.000)\end{array}$ \\
\hline
\end{tabular}

Notes: The regressions are estimated using OLS with t-values produced by robust standard errors in parentheses. The constants are not reported. Observations (N), R-squared (R-sq), F-statistics and the associated p-values are reported. Firm level data are aggregated up to the 14 regions, industries (classified by the SIC-4 primary codes) within $14-$ 
region, and industries within postal area (identified by postal codes) levels respectively for the regional level, region-industry level and postal code-industry level samples. For the regional level regressions, to ensure the reliability of the OLS statistical inference, Network (Putnam), Network (olson), Trust (foreign), Urban and High education are not included so that the number of regressors is less than the number of observations. Significance at the $1 \%, 5 \%$ and $10 \%$ levels are denoted respectively by $* * *, * *$ and *. 
Table 4: Social Capital and Firm Performance: Robustness to Alternative Measures of Firm Performance and 1999 Firm-level Sample

\begin{tabular}{|c|c|c|c|c|}
\hline & \multicolumn{3}{|c|}{1999 Firm-level Sample } & \multirow{2}{*}{$\begin{array}{c}\text { Alternative Measures of Firm } \\
\text { Performance } \\
(4)\end{array}$} \\
\hline & (1) & $(2)$ & (3) & \\
\hline & $\begin{array}{c}\text { Return on } \\
\text { Assets }\end{array}$ & $\begin{array}{c}\text { Current } \\
\text { Ratio }\end{array}$ & Solvency Ratio & Profit Margin \\
\hline \multicolumn{5}{|c|}{ Social Capital and Religion Variables } \\
\hline Trust (domestic) & $\begin{array}{c}1.586 * * * \\
(3.33)\end{array}$ & $\begin{array}{c}0.331 * * * \\
(4.35)\end{array}$ & $\begin{array}{c}2.272 * * \\
(2.25)\end{array}$ & $\begin{array}{l}-2.412 \\
(-1.39)\end{array}$ \\
\hline Trust (foreign) & $\begin{array}{c}0.684^{* * *} \\
(3.20)\end{array}$ & $\begin{array}{c}0.325 * * \\
(2.39)\end{array}$ & $\begin{array}{c}1.788 * * * \\
(8.97)\end{array}$ & $\begin{array}{c}4.302 * * \\
(2.30)\end{array}$ \\
\hline Norm & $\begin{array}{c}0.313^{* * * *} \\
(3.34)\end{array}$ & $\begin{array}{l}0.083 \\
(0.96)\end{array}$ & $\begin{array}{l}0.512 \\
(1.57)\end{array}$ & $\begin{array}{l}0.631 \\
(1.36)\end{array}$ \\
\hline Network (all-group) & $\begin{array}{c}3.857 * * * \\
(3.30)\end{array}$ & $\begin{array}{l}0.336 \\
(0.25)\end{array}$ & $\begin{array}{c}5.809 * * * \\
(7.80)\end{array}$ & $\begin{array}{c}33.742 * \\
(1.92)\end{array}$ \\
\hline Network (Putnam) & $\begin{array}{c}1.942 * * * \\
(2.61)\end{array}$ & $\begin{array}{c}1.209 * * * \\
(2.79)\end{array}$ & $\begin{array}{c}3.085^{* * *} \\
(3.05)\end{array}$ & $\begin{array}{l}-0.835 \\
(-0.48)\end{array}$ \\
\hline Network (Olson) & $\begin{array}{c}2.265^{* * * *} \\
(3.18)\end{array}$ & $\begin{array}{l}-0.339 \\
(-0.59)\end{array}$ & $\begin{array}{c}1.879 * * * \\
(7.11)\end{array}$ & $\begin{array}{c}10.77^{*} \\
(1.73)\end{array}$ \\
\hline \multicolumn{5}{|l|}{ Control Variables } \\
\hline $\begin{array}{c}\text { Firm size (total } \\
\text { assets) }\end{array}$ & $\begin{array}{c}2.11 \mathrm{e}-07 \\
(1.19)\end{array}$ & $\begin{array}{c}-2.68 \mathrm{e}-08 \\
(-0.11)\end{array}$ & $\begin{array}{c}-7.21 \mathrm{e}-07 \\
(-1.37)\end{array}$ & $\begin{array}{c}-9.72 \mathrm{e}-08 \\
(-1.17)\end{array}$ \\
\hline Health status & $\begin{array}{l}-1.674 \\
(-1.28)\end{array}$ & $\begin{array}{c}0.212^{* *} \\
(2.39)\end{array}$ & $\begin{array}{c}6.884 * * * \\
(5.87)\end{array}$ & $\begin{array}{l}-4.183 \\
(-1.53)\end{array}$ \\
\hline High education & $\begin{array}{l}1.527^{*} \\
(1.94)\end{array}$ & $\begin{array}{c}0.453 * * * \\
(4.08)\end{array}$ & $\begin{array}{c}0.823 * * * \\
(2.78)\end{array}$ & $\begin{array}{l}0.458 \\
(0.93)\end{array}$ \\
\hline Urban & $\begin{array}{c}2.564^{* *} \\
(2.40)\end{array}$ & $\begin{array}{l}1.282 \\
(1.60)\end{array}$ & $\begin{array}{c}6.294^{* * *} \\
(1.98)\end{array}$ & $\begin{array}{c}18.946 * * \\
(1.97)\end{array}$ \\
\hline Border & $\begin{array}{c}-5.514 * * * \\
(-2.83)\end{array}$ & $\begin{array}{l}-3.316 \\
(-1.57)\end{array}$ & $\begin{array}{c}-8.792^{* *} \\
(-2.21)\end{array}$ & $\begin{array}{c}-22.018 \\
(1.45)\end{array}$ \\
\hline \multicolumn{5}{|l|}{ Diagnostic Statistics } \\
\hline $\mathrm{N}$ & 149265 & 136497 & 167015 & 7936 \\
\hline $\mathrm{R}-\mathrm{sq}$ & 0.19 & 0.18 & 0.29 & 0.11 \\
\hline $\begin{array}{l}\text { F-statistic } \\
\text { (p-value) }\end{array}$ & $\begin{array}{c}16.53 \\
(0.000)\end{array}$ & $\begin{array}{c}11.12 \\
(0.000)\end{array}$ & $\begin{array}{c}80.18 \\
(0.000)\end{array}$ & $\begin{array}{c}31.34 \\
(0.000)\end{array}$ \\
\hline
\end{tabular}

Notes: The regressions are estimated using OLS with t-values produced by robust standard errors in parentheses. The constants are not reported. Observations (N), R-squared (R-sq), F-statistics and the associated p-values are reported. The 1999 sample regressions include the core firm performance indicators: "Return on Assets", "Current Ratio" and "Solvency Ratio". The Alternative measure of firm performance such as "profit margin" is from the core 2008 sample, they are used in the robustness checks only due to their unideal low observations. Significance at the $1 \%, 5 \%$ and $10 \%$ levels are denoted respectively by $* * *, * *$ and *.

different levels of aggregation is to ensure that our main results are not driven by a specific type of level of aggregation. In other words, we want to show that match regional social capital with firmlevel performance (so that each firm in the same region face the same social capital environment) is not a structure issue in terms of econometric modelling. 
Second, since we have both two years of social capital data (1999 and 2008), corresponding to the firm-level performance data, we decide to nevertheless check if the main results are robust in the 1999 sample. The reason why we choose 2008 over 1999 in the main regressions are two folds: (1) it is more recent, and (2) it fully matches the firm performance data, whereas observations in 1999 for firm performance are very low in our sample, consequently for some observations we use the year 2000 or 2001 as a proxy to match the 1999 social capital data. This is indeed not very ideal, hence the 1999 sample is used for robustness check only. And finally, we also include an alternative measure of firm performance: profit margin, which has very low observations (only around 5\% of the other three measures) itself, hence not ideal to be included in the main regression.

Table 3 shows results from the three aggregation levels, with Columns (1) to (3) showing the results of regional level sample, Columns (4) to (6) for region-industry sample and Columns (7) to (9) giving the results for the postal code-industry sample. In the regional level regressions, due to the natural limitation of low observations, we decide to omit the Trust (foreign), Network (Putnam) and Network (Olson) variables. ${ }^{5}$ Even though the regional level regression is troubled with only 14 observations, from Columns (1) to (3) we nevertheless find evidence that trust (domestic), norm and network (allgroup) can positively influence at least one firm performance indicator. This is also true for the Catholic, Protestant and Buddhist religions, while Jews and Muslims religious groups have no significant impact on firm growth.

Results from the region-industry and postal code-industry sample suggest the same: both social capital and religion are important for firm performance. This in turn implies that the significant and positive effects of social capital and religion on firm performance are universal (in terms of significance) across all industries, so that there's no immediate urgent need to explore industryspecific effects. From Columns (1) to (9), all the social capital and religion (except for Muslim) variables are significant in explaining at least one firm performance indicator at a specific aggregation level. Moreover, comparing R-squares from the two aggregation levels and the firm-level main results, it seems to suggest that both firm-level and postal-code level are ideal models, as they give the highest R-squares with full specification (including all variables in the regression). ${ }^{6}$ This gives us another

\footnotetext{
${ }^{5}$ Trust (domestic) is combined by more trust-related variables as compared with Trust (foreign), where only three variables are combined, hence, represent a more accurate measure of trust. Since there's no major differences in the roles of the Putnam and Olson groups on firm performance, we decide to include only the Network (all-group). Education and Urban dummies as control variables are also omitted to meet the criteria that the number of regressors should be less than the number of observations. Omitting other control variables instead of these two give similar results.

${ }^{6}$ The R-squares from the regional level regressions are unreliable at 0.99 , due to extreme low observations.
} 
level of confidence that our decision to choose the firm-level sample as our core sample is sound, as it gives the best mode-fit and contains the highest observations.

Table 4 gives results on the 1999 sample and the alternative measure of firm performance: profit margin. The 1999 sample, albeit with its natural imperfections, shows consistent results as observed in the 2008 core sample, at least one of the five social capital variables along with Catholic, Protestant and Jews religions shows positive and significant influence on at least one of the firm performance indicators with Muslim and Buddhist being insignificant. The influence of social capital and religion on profit margin, the alternative measure of firm performance is non-surprisingly not strong (due to its low observations) compared with the results from the core regressions. Nevertheless we manage to find out significant results for trust and network on three occasions. In general, the various robustness checks we conduct suggest that our main results are not driven by different aggregation levels (sample structure), are robust to alternative measures of firm performance and different sampling year, and the relationships between social capital, religion and firm performance are evident with even imperfect sampling conditions such as low observations and imperfect matching of data.

\section{Conclusions}

In sum, this paper explores the potential effect of social capital and religion on firm performance by employing a novel approach: to look at social capital and religion environment rather than the narrow aspect of entrepreneurs own social network. Obtaining trust, norm network and religion variables from the Danish and European Value studies and extracting various firm performance indicators from the Orbis and Amadeus database, this paper has found the following:

First, social capital as measured by trust, norm and network have significant positive effects on firm performance as measured by return on asset, current ratio and solvency ratio. The magnitude of these effects are stronger on return on asset and solvency ratio than on current ratio, suggesting social capital is more important for firms' profit and long term liquidity than short term liquidity. These effects are strong after we control for firm size, health status of local residents, border and urban characteristics and local education level.

Second, specifically, trust in domestic institutions seem to matter more for firm performance than trust in foreign institutions, this is not surprising considering the fact that local firm characteristics have more to do with domestic environment than foreign. Networks, regardless types, can positively influence firm performance, albeit the growth enhancing Putnam group networking matters more for firm performance than the special interest oriented Olson group in terms of significance. 
Third, religion as measured by Catholic, Protestant, Jews and Buddhist seem to positively affect firm performance, while Muslim shows no such trait. A possible explanation could be that the Muslim religion exhibits strong exclusivity in terms of the level of communication and cooperation its members are allowed to conduct with people outside the group. This in turn stifles bridging activities, effectively making it a special interest group with no apparent link to firm activities, hence the general insignificance of its role in firm performance.

Moreover, the above results are robust under the regional, region-industry and postal code-industry level of aggregation, suggesting that there're no industry-specific differences in the significance of these effects. Using alternative measure of firm performance (profit margin) and the 1999 sample yield similar results, suggesting overall robust relationship between social capital, religion and firm performance.

\section{References}

Adler, P.S. and Kown, S., 2002, "Social Capital: Prospects for a New Concept", Academy of Management Review, 27(1), 17-40.

Aldrich, H.E. and Zimmer, C., 1986, "Entrepreneurship Through Social Networks. In: Sexton, D.L. and Smilor, R.W.(Eds), the Art and Science of Entrepreneurship", Ballinger Publishing Company, Cambridge, MA.

Barro, R.J. and McCleary, R.M., 2003, "Religion and Economic Growth Across Countries", American Sociological Review, 68(5), 760-781.

Batjargal, B., 2003, "Social Capital and Entrepreneurial Performance in Russia: a Longitudinal Study”, Organization Studies, 24(4), 535-556.

Bhagavatula, S., Elfring, T., van Tilburg, A. and van de Bunt, G.G., 2010, ’How Social and Human Capital Influence Opportunity Recognition and Resource Mobilization in India's handloom Industry", Journal of Business Venturing, 25, 245-260.

Blum, U. and Leonard, D., 2001, “Religion and Economic Growth: Was Weber Right?”, Journal of Evolutionary Economics, 11(2), 207-230.

Dodd, S.D. and Gotsis, G., 2007, “The Interrelationships between Entrepreneurship and Religion”, The International Journal of Entrepreneurship and Innovation, 8(2), 93-104.

Elfring, T. and Hulsink, W., 2003, "Networks in Entrepreneurship: the Case of High-Technology Firms", Small Business Economics, 21, 409-422.

Elfring, T. and Hulsink, W., 2007, "Networking by Entrepreneurs: Patterns of Tie Formation in Emerging Organizations:, Organization Studies, 28(12), 1849-1872.

Galbraith, C.S. and Galbraith, D.M., 2007, “An Empirical Note on Entrepreneurial Activity, Intrinsic Religiosity and Economic Growth", Journal of Enterprising Communities, I(2), 188201.

Greif, A., 1994, "Cultural Beliefs and the Organization of Society: A Historical and Theoretical Reflection on Collectivist and Individual Societies", Journal of Political Economy, 102(5), 912-950.

Hite, J.M. and Hesterly, W.S., 2001, "The Evolution of Firm Networks: from Emergence to Early Growth of the Firm", Strategic Management Journal, 22, 275-286. 
Hoang, H. and Antoncic, B., 2003, "Network-based Research in Entrepreneurship: a Critical Review", Journal of Business Venturing, 18, 165-187.

Hoe, C.H., Isa, F.M., Hin, C.W., Hashim, N., Yunus, J.M. and Abdullah, H.H., 2012, "Development of Women Entrepreneurs: the Case of Malaysia", World Journal of Social Science, 2(6), 123-145.

Knack, S. and Keefer, P., 1997, "Does Social Capital Have an Economic Payoff? A Cross-Country Investigation", The Quarterly Journal of Economics, 112(4), 1251-1288.

Landes, D.S., 1998, The Wealth and Poverty of Nations, New York: W.W. Norton and Company.

Lewis, W.A., 1955, The Theory of Economic Growth, Homewood, IL: Richard D. Irwin.

Martinez, M.A. and Aldrich, H.E., 2011, "Networking Strategies for Entrepreneurs: Balancing Cohesion and Diversity", International Journal of Entrepreneurial Behavior and Research, 17(1), 7-38.

Maurer, L. and Ebers, M., 2006, "Dynamics of Social Capital and Their Performance Implications: Evidence from Biotechnology Start-ups. Administrative Science Quarterly, 51(2), 262-292.

McEvily, B. and Zaheer, A., 1999, "Bridging Ties a Source of Firm Heterogeneity in Competitive Capabilities", Strategic Management Journal, 20, 1133-1156.

Noland, M., 2005, "Religion and Economic Performance", World Development, 33(8), 1215-1232.

Nwankwo, S., Gbadamosi, A. and Ojo, S., 2012, "Religion, Spirituality and Entrepreneurship: The Church as Entrepreneurial Space among British Africans. Society and Business Review, 7(2), 149-167.

Olson, M., 1982, "The Rise and Decline of Nations", New Haven: Yale University Press.

Putnam, R.D. and Leonardi, R. and Nanetti, R.Y., 1993, “Making Democracy Work”, New Jersey: Princeton University Press.

Stam, W., 2010, "Industry Event Participation and Network Brokerage among Entrepreneurial Ventures", Journal of Management Studies, 47(4), 625-653.

Stam, W., Arzlanian, S. and Elfring, T., 2014, "Social Capital of Entrepreneurs and Small Firm Performance: A Meta-Analysis of Contextual and Methodological Moderators", Journal of Business Venturing, 29, 152-173.

Stuart, T.E. and Sorensen, O., 2007, "Strategic Networks and Entrepreneurial Ventures", Strategic Entrepreneurship Journal, 1(3-4), 211-227.

Tu, Q., Bulte, E. and Tan, S., 2011, "Religiousity and Economic Performance: Micro-economic Evidence from Tibetan Area", China Economic Review, 22(1), 55-63.

Weber, M., 1905/2002, The Protestant Ethic and the "Spirit" of Capitalism and Other Writings. New York: Penguin Books.

Wang, C. and Steiner, B., 2015, "Can Ethnolinguistic Diversity Explain Cross-Country Differences in Social Capital?”, Economic Record, DOI: 10.1111/1475-4932.12195 


\section{Appendix}

Table A1 : Social Capital Variables Construction: Constituting Variables and PCA coefficients

\begin{tabular}{|c|c|c|}
\hline Social Capital Variables & Constituting Variables in Danish Value studies & $\begin{array}{l}\text { First PCA } \\
\text { Coefficients }\end{array}$ \\
\hline \multirow[t]{15}{*}{ Trust (domestic) } & $\begin{array}{l}\text { People can be trusted, percentage of respondents answered "yes" are taken for a given region } \\
\text { (Amt) }\end{array}$ & 0.158 \\
\hline & $\begin{array}{l}\text { How much confidence in church, percentage of respondents answered "a great deal and "quite } \\
\text { a lot" are taken for a given region (Amt) }\end{array}$ & 0.012 \\
\hline & $\begin{array}{l}\text { How much confidence in armed forces, percentage of respondents answered "a great deal and } \\
\text { "quite a lot" are taken for a given region (Amt) }\end{array}$ & 0.363 \\
\hline & $\begin{array}{l}\text { How much confidence in education system, percentage of respondents answered "a great deal } \\
\text { and "quite a lot" are taken for a given region (Amt) }\end{array}$ & 0.268 \\
\hline & $\begin{array}{l}\text { How much confidence in the press, percentage of respondents answered "a great deal and } \\
\text { "quite a lot" are taken for a given region (Amt) }\end{array}$ & 0.303 \\
\hline & $\begin{array}{l}\text { How much confidence in trade unions, percentage of respondents answered "a great deal and } \\
\text { "quite a lot" are taken for a given region (Amt) }\end{array}$ & 0.159 \\
\hline & $\begin{array}{l}\text { How much confidence in the police, percentage of respondents answered "a great deal and } \\
\text { "quite a lot" are taken for a given region (Amt) }\end{array}$ & 0.343 \\
\hline & $\begin{array}{l}\text { How much confidence in parliament, percentage of respondents answered "a great deal and } \\
\text { "quite a lot" are taken for a given region (Amt) }\end{array}$ & 0.033 \\
\hline & $\begin{array}{l}\text { How much confidence in civil service, percentage of respondents answered "a great deal and } \\
\text { "quite a lot" are taken for a given region (Amt) }\end{array}$ & 0.262 \\
\hline & $\begin{array}{l}\text { How much confidence in social security system, percentage of respondents answered "a great } \\
\text { deal and "quite a lot" are taken for a given region (Amt) }\end{array}$ & 0.266 \\
\hline & $\begin{array}{l}\text { How much confidence in health care system, percentage of respondents answered "a great } \\
\text { deal and "quite a lot" are taken for a given region (Amt) }\end{array}$ & 0.396 \\
\hline & $\begin{array}{l}\text { How much confidence in justice system, percentage of respondents answered "a great deal } \\
\text { and "quite a lot" are taken for a given region (Amt) }\end{array}$ & 0.260 \\
\hline & $\begin{array}{l}\text { How much confidence in major companies, percentage of respondents answered "a great deal } \\
\text { and "quite a lot" are taken for a given region (Amt) }\end{array}$ & 0.279 \\
\hline & $\begin{array}{l}\text { How much confidence in environmental organizations, percentage of respondents answered } \\
\text { "a great deal and "quite a lot" are taken for a given region (Amt) }\end{array}$ & 0.009 \\
\hline & $\begin{array}{l}\text { How much confidence in political parties, percentage of respondents answered "a great deal } \\
\text { and "quite a lot" are taken for a given region (Amt) }\end{array}$ & 0.149 \\
\hline
\end{tabular}


Trust (foreign)

Norm

\section{Network (Putnam group)}

"quite a lot" are taken for a given region (Amt)

How much confidence in European Union, percentage of respondents answered "a great deal and "quite a lot" are taken for a given region (Amt)

How much confidence in Nato, percentage of respondents answered "a great deal and "quite a lot" are taken for a given region (Amt)

How much confidence in UN, percentage of respondents answered "a great deal and "quite a lot" are taken for a given region (Amt)

Do you justify: cheating on tax, percentage of respondents that answered "never" are taken for a given region (Amt)

Do you justify: taking illegal drugs, percentage of respondents that answered "never" are taken for a given region (Amt)

Do you justify: lying in own interest, percentage of respondents that answered "never" are taken for a given region (Amt)

Do you justify: adultry, percentage of respondents that answered "never" are taken for a given region (Amt)

Do you justify: accepting a bribe, percentage of respondents that answered "never" are taken for a given region (Amt)

Do you justify: paying cash to avoid taxes, percentage of respondents that answered "never" are taken for a given region (Amt)

Do you justify: avoiding fare on public transportation, percentage of respondents that answered "never" are taken for a given region (Amt)

Do you justify: illegal prostitution, percentage of respondents that answered "never" are taken for a given region (Amt)

Do you justify: experiments on human embryos, percentage of respondents that answered "never" are taken for a given region (Amt)

Do you justify: manipulating food, percentage of respondents that answered "never" are taken for a given region (Amt)

Do you justify: death penalty, percentage of respondents that answered "never" are taken for a given region (Amt)

Do you justify: drunk driving, percentage of respondents that answered "never" are taken for a given region (Amt)

Do you belong to religious organization, percentage of respondents answered "yes" in a given region (Amt) are taken.

Do you belong to cultural activities, percentage of respondents answered "yes" in a given region (Amt) are taken.
0.516

0.640

0.568

0.162

0.430

0.386

0.062

0.355 0.121

0.610 0.469 
given region (Amt) are taken.

Do you belong to Sports/recreation organization, percentage of respondents answered "yes"

in a given region (Amt) are taken.

Do you belong to education organization, percentage of respondents answered "yes" in a given

Network (Olson group) region (Amt) are taken.

Do you belong to trade union, percentage of respondents answered "yes" in a given region

(Amt) are taken.

Do you belong to political parties/groups, percentage of respondents answered "yes" in a

0.568

given region (Amt) are taken.

Do you belong to development/human rights, percentage of respondents answered "yes" in a

0.620 given region (Amt) are taken.

Do you belong to professional associations, percentage of respondents answered "yes" in a

0.536 given region (Amt) are taken.

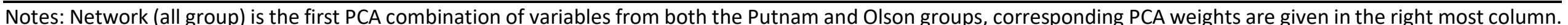

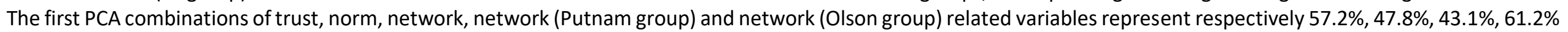

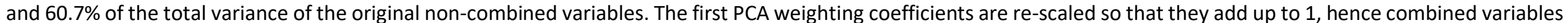
(trust, norm, network (all group), network (Putnam), network (Olson)) also fall into the 0 to 100 percentage-point range. 
Table A2: Correlation Between Main Variables (2008 firm-level sample)

\begin{tabular}{|c|c|c|c|c|c|c|c|c|c|c|c|c|c|c|}
\hline & $\begin{array}{c}\text { Return } \\
\text { on } \\
\text { Asset }\end{array}$ & $\begin{array}{l}\text { Current } \\
\text { Ratio }\end{array}$ & $\begin{array}{c}\text { Solvency } \\
\text { Ratio }\end{array}$ & $\begin{array}{c}\text { Trust } \\
\text { (domestic) }\end{array}$ & $\begin{array}{c}\text { Trust } \\
\text { (foreign) }\end{array}$ & Norm & $\begin{array}{l}\text { Network } \\
\text { (all group) }\end{array}$ & $\begin{array}{l}\text { Network } \\
\text { (Putnam) }\end{array}$ & $\begin{array}{l}\text { Network } \\
\text { (Olson) }\end{array}$ & $\begin{array}{c}\text { Firm size } \\
\text { (total assets) }\end{array}$ & $\begin{array}{l}\text { Health } \\
\text { status }\end{array}$ & $\begin{array}{c}\text { High } \\
\text { education }\end{array}$ & Urban & Border \\
\hline $\begin{array}{c}\text { Return on } \\
\text { Asset }\end{array}$ & 1 & & & & & & & & & & & & & \\
\hline $\begin{array}{c}\text { Current } \\
\text { Ratio }\end{array}$ & -0.02 & 1 & & & & & & & & & & & & \\
\hline $\begin{array}{c}\text { Solvency } \\
\text { Ratio }\end{array}$ & -0.01 & 0.47 & 1 & & & & & & & & & & & \\
\hline $\begin{array}{c}\text { Trust } \\
\text { (domestic) }\end{array}$ & 0.18 & 0.15 & 0.14 & 1 & & & & & & & & & & \\
\hline $\begin{array}{c}\text { Trust } \\
\text { (foreign) }\end{array}$ & 0.11 & 0.14 & 0.10 & 0.18 & 1 & & & & & & & & & \\
\hline Norm & 0.07 & 0.16 & 0.24 & 0.28 & 0.27 & 1 & & & & & & & & \\
\hline $\begin{array}{l}\text { Network } \\
\text { (all-group) }\end{array}$ & 0.11 & 0.18 & 0.19 & 0.11 & 0.13 & 0.40 & 1 & & & & & & & \\
\hline $\begin{array}{l}\text { Network } \\
\text { (Putnam) }\end{array}$ & 0.11 & 0.10 & 0.17 & 0.11 & 0.49 & 0.02 & 0.41 & 1 & & & & & & \\
\hline $\begin{array}{l}\text { Network } \\
\text { (Olson) }\end{array}$ & 0.11 & 0.10 & 0.17 & 0.11 & 0.40 & 0.20 & 0.89 & 0.65 & 1 & & & & & \\
\hline $\begin{array}{l}\text { Firm size } \\
\text { (total } \\
\text { assets) }\end{array}$ & -0.00 & -0.00 & -0.00 & -0.00 & 0.00 & 0.00 & 0.00 & 0.00 & 0.00 & 1 & & & & \\
\hline $\begin{array}{l}\text { Health } \\
\text { status }\end{array}$ & 0.19 & 0.21 & 0.14 & -0.15 & 0.01 & 0.01 & 0.33 & -0.02 & 0.13 & 0.00 & 1 & & & \\
\hline $\begin{array}{c}\text { High } \\
\text { education }\end{array}$ & 0.16 & 0.17 & 0.10 & -0.58 & 0.02 & 0.01 & 0.32 & 0.31 & 0.33 & 0.00 & 0.60 & 1 & & \\
\hline Urban & 0.09 & 0.15 & 0.03 & -0.11 & 0.57 & -0.43 & 0.06 & -0.17 & -0.12 & 0.00 & 0.55 & 0.54 & 1 & \\
\hline Border & -0.08 & -0.09 & -0.07 & 0.32 & 0.36 & -0.07 & -0.47 & -0.46 & -0.55 & -0.00 & -0.39 & -0.31 & 0.23 & 1 \\
\hline
\end{tabular}

